\title{
Cambios psicosociales en un programa de intervención con hombres penados por violencia contra la mujer
}

\author{
Psychosocial changes in an intervention program with \\ intimate partner violence offenders
}

\author{
Raquel Conchell Diranzo ${ }^{1}$ \\ Marisol Lila Murillo ${ }^{2}$ \\ Alba Catalá Miñana $a^{3}$
}

\section{Resumen}

En este trabajo se presentan los resultados obtenidos en relación a la evaluación de la eficacia de un programa de intervención para hombres condenados por violencia contra la mujer, desarrollado en medio abierto en la provincia de Valencia (Programa Contexto). La muestra está compuesta por 109 hombres. Se utilizó un diseño preexperimental con medición pre y post tratamiento. Según los resultados obtenidos, se producen cambios significativos a nivel actitudinal, reduciéndose las actitudes de culpabilización de la víctima. Por otra parte, se producen cambios relativos a variables psicológicas, experimentando los participantes una reducción de la sintomatología depresiva y, por último, a nivel social se incrementa la participación en la comunidad. Se comentan las implicaciones de este estudio para investigaciones futuras.

Palabras clave: cambio, eficacia, Programa Contexto, programas de intervención con maltratadores.

1 Departamento de Psicología Social. Universidad de Valencia. Valencia, España. E-mail: Raquel. Conchell@uv.es. Autora responsable.

2 Departamento de Psicología Social. Universidad de Valencia. Valencia, Espańa. E-mail: Marisol. Lila@uv.es

3 Departamento de Psicología Social. Universidad de Valencia. Valencia, Espańa. E-mail: Alba.Catala@uv.es 


\section{Abstract}

This work presents the results obtained in relation to the evaluation of the effectiveness of an intervention program for men convicted of violence against women, developed in an open environment in the province of Valencia, Spain (Context Program). The sample consists in 109 men. It consists in a preexperimental design, with pre and post treatment measurement. According to the results, significant changes in attitude level have appeared, reducing the attitudes of blaming the victim. On the other hand, changes related to psychological variables are observed, as participants experienced a reduction of depressive symptoms and also showed increased community participation, at the social level. The implications of these findings are discussed regarding future research.

Key words: change, effectiveness, context program, batterers intervention programs.

En los últimos años se ha visto incrementado, en todo el mundo, el interés de investigadores y profesionales por la implementación de programas de intervención con maltratadores (Echeburúa \& FernándezMontalvo, 2009). En España, las primeras experiencias de intervención e investigación se desarrollaron en prisión con hombres condenados por cometer un delito grave de violencia de género en el País Vasco (España) en 1995, experiencia liderada por Enrique Echeburúa. La aprobación y entrada en vigor de la Ley Orgánica 1/2004, de Medidas de Protección Integral contra la Violencia de Género en España, ha traído consigo un gran número de sentencias que contemplan la suspensión de la pena condicionada a la participación en programas de intervención con maltratadores, lo que ha supuesto un aumento de dichos programas en medio abierto.

En España, no existe consenso acerca de lo que constituye la violencia contra la mujer ni contamos con un concepto unitario que la defina. Unas veces se habla de violencia de género, para indicar que el motivo 
principal de esta conducta es el mantenimiento de las desigualdades entre hombres y mujeres, otras de violencia masculina contra las mujeres, con el fin de destacar la distribución de género entre víctimas y agresores, etc. Por ello, en este trabajo se utiliza indistintamente durante todo el texto ambos términos.

Hay que tener en cuenta varias razones sobre la importancia de evaluar de forma adecuada si cumplen su cometido los programas de intervención con maltratadores, es decir, si son eficaces (Bennett \& Williams, 2001). En primer lugar, hay un deseo por parte de la sociedad de confiar en la eficacia de este tipo de programas. En segundo lugar, las mujeres víctimas de violencia de género con frecuencia siguen manteniendo relaciones con los maltratadores, por lo que la eficacia de los programas se convierte en una cuestión de seguridad personal, ya que pueden crear falsas expectativas. Por último, las personas que trabajan en dichos programas están interesadas en los resultados, para poder mejorar el nivel de eficacia de ellos.

Por todo esto, es importante que los programas de intervención sean eficaces, entendiendo el término eficacia como la capacidad de lograr el efecto que se desea o se espera (Real Academia Española, 2012). Existe una controversia en torno a la eficacia de los programas de intervención con maltratadores (Babcock \& LaTaillade, 2000; Dunford, 2000). Las primeras evaluaciones indicaban una alta consistencia de tasas de éxito, aunque los resultados podían estar reflejando deficiencias metodológicas de la investigación, en lugar de una eficacia real en la reducción de la violencia (Chen, Bersani, Myers \& Denton, 1989; Ford \& Regoli, 1993; Gondolf, 1987). Posteriormente se llevaron a cabo evaluaciones más rigurosas y, a diferencia de los estudios anteriores, se obtuvieron resultados ambiguos sobre la eficacia de los programas de intervención con maltratadores (Babcock, Green \& Robie, 2004; Eckhardt, Murphy, Black \& Suhr, 2006; Feder \& Forde, 2000; Feder \& Wilson, 2005; Feder, Wilson \& Austin, 2008; ). Estos resultados, posiblemente, reflejan las diferencias en el rigor metodológico utilizado (Feder et al., 2008). 
En contraste, diversas investigaciones indican que los hombres penados por violencia contra la mujer que asisten a un programa de intervención ofrecen resultados favorables, obteniendo una disminución significativa de las tasas de reincidencia respecto a la violencia contra la mujer (Coulter \& VandeWeerd, 2009; Cranwell, Kolodinsky, Carsten, Schmidt, Larson \& MacLachlan, 2007; Echeburúa \& Fernández-Montalvo, 2009; Echeburúa, Fernández-Montalvo \& Amor, 2006; Gondolf, 2002; Taylor, Davies \& Maxwell, 2001).

En España, respecto a los programas de intervención con maltratadores, existen escasas evaluaciones, pero la mayoría de ellas han obtenido resultados prometedores (Echeburúa \& Del Corral, 1998). No cabe duda de la necesidad de seguir realizando más estudios que arrojen luz en relación a la eficacia de la intervención psicológica con hombres penados por violencia contra la mujer, reduciendo las conductas de maltrato y evitando la reincidencia (Echeburúa, Sarasua, Zubizarreta \& Corral, 2009; Feder et al., 2008; Lorente, 2001).

Una de las formas más utilizadas para evaluar la eficacia de un programa de intervención con maltratadores es la reincidencia (Coulter \& VandeWeerd, 2009; Echeburúa \& Fernández-Montalvo, 2009). Sin embargo, la evaluación del cambio que se produce en variables psicosociales relevantes, consideradas como factores protectores/de riesgo en relación a la violencia contra la mujer en las relaciones de pareja, son datos muy importantes que ayudan a medir la eficacia de la intervención y, a la vez, disminuir la reincidencia (Arce \& Farińa, 2010). Este es el caso de las actitudes hacia la violencia contra la pareja, la asunción de responsabilidad, algunas variables psicológicas como la autoestima o los síntomas depresivos y el apoyo social percibido.

Por ejemplo, numerosas investigaciones han relacionado determinadas actitudes y creencias en torno a la violencia en las relaciones de pareja con una mayor tolerancia de la misma y, por lo tanto, con un incremento de las probabilidades de utilizar la violencia en la relación 
(Gracia \& Herrero, 2006a, 2006b). Hay que tener en cuenta que si algunos incidentes de violencia de género son percibidos como aceptables o no suficientemente graves (e.g., amenazas, agresiones físicas no severas) es menos probable que aparezcan actitudes positivas hacia la intervención (Gracia, Herrero, Lila \& Fuente, 2010) o hacia el cambio de conducta de los penados.

En cuanto a la asunción de responsabilidad de los hechos ocurridos por parte del agresor, se considera un elemento clave para afrontar las consecuencias de su conducta, garantizar que los maltratadores asistan continuadamente y lleguen a finalizar los programas de intervención a los que son derivados desde las instancias judiciales, aspecto importante relacionado con la eficacia y reincidencia (Daly \& Pelowski, 2000; Medina, 2002).

Por otro lado, los programas de intervención trabajan multitud de variables psicológicas (control de impulsos, ira, celos, etc.). Uno de los síntomas de desajuste psicológico que se ha encontrado vinculado a las características de los maltratadores son los síntomas depresivos (Maiuro, Cahn, Vitaliano, Wagner \& Zegree, 1988). Otra de ellas es la autoestima (Goldstein \& Rosenbaum, 1985; Steel \& Pollack, 1974; Walker, 1984), características que se han visto asociadas a la frecuencia y severidad en la violencia de género (Cascardi \& O’Leary, 1992).

Finalmente, el concepto de apoyo social ha ocupado un lugar central en el análisis de los determinantes sociales de la salud y el bienestar, y está estrechamente vinculado al ámbito de la intervención comunitaria (Cohen, Gottlieb \& Underwood, 2000; Cowen, 2000; Eckersley, Dixon \& Douglas, 2001; Gracia, 1997; Gracia \& Herrero, 2006b; Montero, 2003). Asimismo, otras fuentes señalan también la importancia de la influencia social respecto a hombres maltratadores, planteando que la permanencia del participante en un ambiente socio-comunitario antisocial (p.ej., en una comunidad con elevados contenidos y actitudes sexistas) o incompetente socialmente, aumenta las probabilidades de reincidencia (Gracia, Herrero, Lila \& Fuente, 2009). 
El objetivo de este estudio será evaluar si se producen cambios significativos como consecuencia de la participación en un programa de intervención para hombres condenados por violencia de género (Programa Contexto), en variables relacionadas con este tipo de violencia y tratadas específicamente en el programa de intervención. Para ello se tomarán medidas de un conjunto de variables psicosociales antes del inicio de la intervención (Pretratamiento: Tiempo 1), variables que volverán a medirse tras finalizar la intervención (Postratamiento: Tiempo 2).

En relación al objetivo general de este estudio, las principales hipótesis que nos planteamos en nuestro trabajo son cuatro:

- La intervención producirá cambios en actitudes relacionadas con la percepción de la violencia de género, de forma que en Tiempo 2 serán mayores los niveles de gravedad percibida de las situaciones de violencia de género y la intención de denuncia en casos de violencia de género, así como menores los niveles de tolerancia hacia la violencia y de culpabilización de la victima.

- La intervención generará cambios en los estilos de atribución de responsabilidad de los penados, de forma que se producirán más atribuciones relacionadas con la asunción de responsabilidad por sus propios actos y menos atribuciones que sitúen la culpa en terceras personas (la víctima) o agentes externos (la ley, la sociedad, el alcohol, etc.).

- La intervención producirá cambios en variables de ajuste psicológico (sintomatología depresiva, autoestima), de forma que, en Tiempo 2 serán menores los niveles de sintomatología depresiva y mayores los niveles de autoestima.

- La intervención producirá un incremento del apoyo social percibido de los penados de forma que, en Tiempo 2, serán mayores los niveles de integración, participación, apoyo formal y apoyo informal percibido. 


\section{Método}

\section{Participantes}

La muestra de este estudio está compuesta por 109 hombres condenados por violencia contra la mujer en las relaciones de pareja, que en la actualidad han finalizado la intervención y que fueron remitidos por vía judicial al Programa Contexto (Programa de Investigación, Formación e Intervención para Hombres Penados por Violencia contra la Mujer en la Provincia de Valencia).

Para la selección de la muestra, así como para la admisión en el programa de intervención, los participantes deben de cumplir unos requisitos, siendo los criterios de inclusión: ser mayor de 18 ańos; tener una suspensión de condena por violencia contra la mujer y la obligatoriedad de participar de un programa de intervención; no tener trastornos de personalidad o psicopatologías graves; no sufrir grave adicción al alcohol u otras sustancias; y no presentar conductas agresivas que puedan poner en peligro la integridad física de los profesionales o compañeros.

El rango de edad comprende de los 20 a los 75 años $(M=38.6, D T$ $=12.11)$. En cuanto al país de nacimiento, la tasa de participantes nacidos en España es 57.8\%, siendo el resto de participantes inmigrantes (42.2\%). De estos últimos, la mayoría (73.9\%) se encuentran en una situación regularizada. El 39.5\% de los participantes están casados. Respecto al nivel de estudios, casi la mitad de la muestra tiene estudios elementales o primarios (44\%) y solamente el $9.2 \%$ tiene estudios universitarios. En cuanto al estatus laboral, el 56\% de los participantes trabaja. Respecto al tipo de contrato, solo un $15.6 \%$ tiene contrato fijo. Si observamos los ingresos anuales, los participantes cuyos niveles de ingresos anuales son menores a 1.800 euros representan un $23.9 \%$ del total de la muestra (Tabla 1 ). 
Tabla 1.

Distribución de las variables sociodemográficas $(N=109)$.

\begin{tabular}{ll}
\hline Variables & Porcentajes \\
\hline País de Nacimiento & \\
$\quad$ Españoles & $57.8 \%$ \\
Inmigrantes & $42.2 \%$ \\
& \\
Situación Regularizada (Sí) & $73.9 \%$ \\
& \\
Estado Civil & \\
Casado & $39.5 \%$ \\
$\quad$ Soltero & $22 \%$ \\
Separado/Divorciado & $37.6 \%$ \\
Viudo & $0.9 \%$
\end{tabular}

Nivel de Estudios

Sin Estudios

$9.2 \%$

Elementales

$44 \%$

Secundarios

$37.6 \%$

Universitarios

$9.2 \%$

Estatus Laboral (Con trabajo)

$56 \%$

Tipo de Contrato

Fijo

$15.6 \%$

Temporal con contrato

$20.2 \%$

Temporal sin contrato

$11 \%$

Autónomo

$10.1 \%$

Otros

$43.1 \%$

Ingresos Anuales
$-1800$
$23.9 \%$
1800-12000
$36.7 \%$
12000-36000
$35.8 \%$
36000-120000
$3.7 \%$

Edad

Media

38.6 


\section{Instrumentos}

A continuación se presentan los instrumentos utilizados en la evaluación de los participantes del programa. El criterio de selección ha sido, por una parte, utilizar pruebas específicas en la evaluación de hombres penados por violencia contra la mujer en lengua castellana y, por otra, instrumentos lo más breves y fiables.

\section{Actitudes hacia la Violencia de Género.}

Escala de Gravedad Percibida de la violencia contra la mujer en las relaciones de pareja (Gracia, García \& Lila, 2008). Escala compuesta por ocho escenarios hipotéticos de violencia contra la mujer en las relaciones de pareja, que el penado tiene que valorar en función de su gravedad. Los escenarios están diseñados de forma que difieren en la severidad de la violencia. Incluyen violencia psicológica (“Una mujer es despreciada y humillada continuamente por su pareja”); amenazas ("Una pareja discute, el hombre insulta a la mujer y amenaza con pegarle"); diferentes grados de violencia física ("En una discusión, un hombre pega a la mujer y después le pide perdón”); así como ejemplos de reincidencia ("Una mujer es golpeada frecuentemente por su pareja, causándole a veces pequeñas lesiones y hematomas, aunque no quiere denunciar los hechos"). Se contesta en una escala de 0 (no es grave en absoluto) a 10 (la situación es muy grave). El índice de consistencia interna es de .94. A mayor puntuación, mayor gravedad percibida de la situación.

Frecuencia Percibida (Gracia \& Herrero, 2006b). Ítem adaptado del Eurobarómetro 51.0 (Comisión Europea, 1999). Se utiliza la siguiente pregunta: "En su opinión, ¿cuál es la frecuencia de la violencia doméstica contra la mujer en la sociedad española?". Las categorías de respuesta son: 1 = nada frecuente, 2 = algo frecuente, 3 = frecuente, y $4=$ muy frecuente.

Culpabilización de la víctima (Gracia \& Herrero, 2006b). Ítem adaptado del Eurobarómetro 51.0 (Comisión Europea, 1999). Los participantes tenían que mostrar su acuerdo o desacuerdo con la siguiente afir- 
mación: "Una causa de la violencia de pareja contra la mujer es la conducta provocativa de la mujer" $(1=s i, 0=n o)$.

Aceptabilidad (Gracia \& Herrero, 2006b). Ítem adaptado del Eurobarómetro 51.0 (Comisión Europea, 1999). Se utiliza la siguiente pregunta: "En su opinión, la violencia doméstica contra la mujer es". Las categorías de respuesta son: 1 = inaceptable en todas las circunstancias y siempre punible, 2 = inaceptable en todas las circunstancias pero no siempre punible, 3 = aceptable en algunas circunstancias y $4=$ aceptable en todas las circunstancias.

Tolerancia hacia la violencia (Gracia \& Herrero, 2006b). Ítem adaptado del Eurobarómetro 51.0 (Comisión Europea, 1999). La pregunta en este caso es: “¿En qué circunstancias considera que una mujer víctima de violencia por parte de su pareja debería denunciarlo a las autoridades?”. Las categorías de respuesta son: 1 = tan pronto se sienta amenazada por su pareja aunque no haya agresiones físicas, 2 = tan pronto haya una agresión aunque no sea grave, y 3 = solo cuando haya una agresión grave.

Intención de denuncia en casos de violencia de género (Gracia \& Herrero, 2006b). Ítem adaptado del Eurobarómetro 51.0 (Comisión Europea, 1999). Esta variable es evaluada con la siguiente pregunta: “¿Qué haría si estando en casa usted oye que un vecino está golpeando a su mujer?" ( 1 = lo denunciaría a la policía, 2 = No lo denunciaría, pero hablaría con ellos, 3 = No haría nada, porque no es asunto mío, $4=$ No lo sé).

\section{Estilos de Atribución de Responsabilidad.}

Escala de Atribución de Responsabilidad (Lila, Herrero \& Gracia, 2008). Escala de ocho ítems para evaluar dónde sitúan los participantes penados por violencia contra la mujer la culpa de la situación que los llevó a ser condenados. Se trata de una escala de respuesta de 100 puntos (0-99). El coeficiente de consistencia interna para la escala total es de .70. La escala está compuesta por tres factores: culpabilización de la víctima, que evalúa el grado en que el participante sitúa la culpa de su situación en las mentiras y/o en características de personalidad o conducta de la víctima ("Me 
encuentro en esta situación por culpa de una falsa denuncia", "Estoy aquí por las mentiras y exageraciones de mi pareja” y "El carácter agresivo, falta de control, nerviosismo o problemas psicológicos de mi pareja es la causa de que me encuentre en esta situación”) $(\alpha=.60)$. A mayor puntuación mayor es la creencia de que la situación que les llevó a ser condenados por violencia de género ha sido responsabilidad de la propia víctima. Defensa Propia, que evalúa el grado en que el participante afirma que su conducta únicamente se ha producido como respuesta a una agresión previa ("Me encuentro en esta situación por haber actuado en defensa propia" y "Estoy aquí por haberme defendido de las agresiones de mi pareja”) ( $\alpha=.74)$. A mayor puntuación mayor es la creencia de que la situación que les llevó a ser condenados por violencia de género ha sido por actuar en defensa propia. Auto-atribución de culpa, está compuesta por 3 ítems que evalúan el grado en que el participante atribuye la causa de los hechos a su propia forma de ser o a problemas personales ("La bebida o uso de otras drogas es la causa de que me encuentre en esta situación", "Mis celos son la causa de que me encuentre en esta situación” y "Mi forma de ser-carácter agresivo, impulsividad, falta de control, nerviosismo, problemas psicológicos, etc.es la causa de que me encuentre en esta situación”) $(\alpha=.62)$. A mayor puntuación mayor es la creencia de que la situación que les llevó a ser condenados por violencia de género ha sido responsabilidad de alguna variable que les ha afectado a ellos y a su conducta (el alcohol, mi impulsividad, mis celos, etc.).

Escala de Minimización (Lila et al., 2008). Esta escala de 4 ítems evalúa el grado en que los participantes restan importancia a los hechos por los que han sido condenados ("La causa de que esté aquí es que la ley se mete en asuntos que son privados", "Me encuentro en esta situación por hacer lo mismo que he visto hacer en mi familia", "Estoy en esta situación por hacer algo que para mis familiares no tiene importancia" y "La causa de que esté aquí es que se le llama violencia contra la pareja a cualquier cosa”). Se trata de una escala de respuesta de 100 puntos (0-99). Su consistencia interna es moderada $(\alpha=.60)$. A mayor puntuación mayor es la creencia de que la situación que les llevó a ser condenados por violencia de género no ha sido grave o no tiene importancia. 


\section{Variables de Ajuste Psicológico.}

Sintomatología Depresiva CESD-7 (Herrero \& Gracia, 2007). Se trata de una versión breve del CES-D (20 ítems) (Radloff, 1977) compuesta por los siete ítems más eficaces para diferenciar las personas deprimidas de las no deprimidas. Pretende identificar niveles elevados de sintomatología depresiva en personas sin diagnóstico clínico. Se trata de detectar el nivel de riesgo de padecer depresión, no para diagnosticarla. Los factores están compuestos por los síntomas más comunes que aparecen en la depresión y el participante debe indicar con qué frecuencia le ha ocurrido en la última semana: (ej.: "Sentía como si no pudiera quitarme de encima la tristeza", "Me parecía que todo lo que hacía era un esfuerzo", "Me costaba concentrarme en lo que estaba haciendo"). Se contesta en una escala de respuesta tipo Likert de 4 puntos, donde 1 es "rara vez o nunca (menos de un día)" y 4 significa "todo el tiempo o la mayoría del tiempo (5-7 días)". La consistencia interna es de .82. A mayor puntuación, mayor riesgo de padecer depresión.

Escala de Autoestima (Rosemberg, 1965). Escala de diez ítems para evaluar la autoestima global, referida a sentimientos globales de aprecio y aceptación de uno mismo (ej., "En general, estoy satisfecho conmigo mismo", "A veces pienso que no sirvo para nada"). Se responde en una escala tipo Likert de cuatro puntos $(1=$ totalmente en desacuerdo; 4 = totalmente de acuerdo). La fiabilidad test-retest es de $.85 \mathrm{y}$ el coeficiente alfa de consistencia interna es de .92. A mayor puntuación, mayor autoestima.

Cuestionario de Autoestima AUT-17 (Gracia, Herrero \& Musitu, 2002). Escala de 17 ítems que evalúa la autoestima del participante. La escala está compuesta por cinco dimensiones. La primera dimensión mide la Autoestima Familiar, el grado de satisfacción con las relaciones que tiene el participante de sus relaciones familiares (ej.: "Me siento muy querido en mi familia"). La segunda dimensión mide la Autoestima Social, la percepción del individuo respecto a su capacidad para crear y mantener relaciones sociales duraderas, así como el grado de satisfacción con sus relaciones actuales (ej.: "Pierdo fácilmente amigos"). La tercera dimensión mide la Autoestima emocional, la percepción de hasta qué punto es capaz 
de controlar sus emociones y si se considera emocionalmente equilibrado (ej.: "soy equilibrado emocionalmente"). La cuarta dimensión mide la Autoestima intelectual, que es la percepción del participante respecto a su funcionamiento intelectual, funciones tales como memoria, concentración, etc. (ej.: "Tengo mala memoria”) y la quinta dimensión, Autoestima física, es la percepción del participante de su cuerpo en términos de aceptación, resistencia y salud (ej.: "Tengo una salud excelente"). También encontramos un índice de Autoestima global que es la suma de todos los anteriores. Se responde en una escala Likert de cinco puntos donde 1 significa "muy en desacuerdo" y 5 quiere decir que está "muy de acuerdo". El índice de consistencia interna es de .78. A mayor puntuación, mayor nivel de autoestima. En nuestra investigación solo utilizaremos las escalas de Autoestima Familiar y Autoestima Social.

\section{Variables Contextuales.}

Apoyo Social Comunitario ASC (Gracia, Herrero \& Musitu, 2002). Escala de 18 ítems que evalúa el apoyo que el participante percibe por parte de la comunidad, del entorno en el que vive. La escala está compuesta por cuatro dimensiones en tres escalas: la primera escala mide Integración Comunitaria (ej.: "me siento identificado con mi comunidad") y Participación en la Comunidad (ej.: "Colaboro en las organizaciones y asociaciones de mi comunidad"). La segunda escala mide Apoyo Social en los Sistemas Informales: asociaciones de vecinos, parroquia, agrupaciones políticas, asociaciones deportivas, etc. (ej.: "En estas organizaciones podría encontrar personas que me ayudaran a resolver mis problemas"); y la escala tres mide Apoyo Social en los Sistemas Formales: centros educativos, centros de rehabilitación, etc. (ej.: "Si tuviera problemas - personales, familiares, etc.- podría encontrar personas en estas organizaciones que me ayudarían a resolverlos"). Se trata de una escala tipo Likert de cinco puntos, donde 1 significa "totalmente en desacuerdo" y 5 supone estar "totalmente de acuerdo". La consistencia interna de la escala 1 es .88, de la escala 2 es .86 y de la escala 3 es .85. A mayor puntuación obtenida en el cuestionario mayor apoyo comunitario percibido. 


\section{Programa de intervención}

El Programa Contexto atiende a hombres condenados por violencia contra la mujer a los que se les remite desde Servicios Sociales Penitenciarios con la condición de la realización de "programas específicos de reeducación y tratamiento psicológico" (LO 1/2004, Título IV, Artículo 35) para la suspensión de la pena de prisión. El Programa se desarrolla bajo la dirección de un equipo de investigación perteneciente al Departamento de Psicología Social de la Universidad de Valencia en estrecha colaboración con la Dirección del Centro de Inserción Social de Picassent (Valencia). Este programa, basado en el modelo Ecológico (Bronfenbrenner, 1979), tiene como objetivo central analizar e intervenir en los principales factores de riesgo y factores protectores existentes en cuatro niveles de análisis (individual, interpersonal, situacional y macrosocial) con el fin de facilitar el cambio de conductas y actitudes hacia la mujer y prevenir futuras conductas violentas con su pareja e hijos en hombres condenados por violencia contra la mujer. El programa se estructura en tres fases: evaluación, intervención y seguimiento. La fase de evaluación comienza con la recepción de los participantes y finaliza con su entrada, si cumple los criterios de inclusión. La fase de intervención tiene una duración aproximada de un año. Está estructurada en siete módulos con sus correspondientes actividades. En el primer módulo se presta atención al conocimiento del grupo y a las normas estipuladas durante toda la intervención. El segundo módulo incluye principios básicos sobre violencia de género, asunción de responsabilidad y mecanismos de defensa. El tercer módulo trabaja aspectos individuales como el autoconcepto, la autoestima, las emociones y las estrategias de autocontrol. Los contenidos en el cuarto módulo están relacionados con variables interpersonales y se incluye el conocimiento de habilidades de comunicación y solución de problemas en la pareja y en los hijos. En el quinto módulo se trabajan las variables situacionales como el apoyo social, el contexto comunitario y el contexto laboral. El módulo sexto se centra en variables socioculturales tales como los roles de género y la mujer en la cultura. Por último, el módulo siete trabaja la prevención de recaídas. La dinámica de trabajo utilizada es de grupo (10-12 personas), conducido 
por dos profesionales formados en violencia de género, que a la vez tienen asignado un supervisor. La última fase del programa es la fase de seguimiento, se realiza cada tres meses vía telefónica y cada seis meses de forma presencial, durante un período de 18 meses.

\section{Procedimiento}

Los participantes son remitidos a través de los Servicios Sociales Penitenciarios a las instalaciones que el Programa tiene en la Universidad de Valencia en grupos de 10-12 participantes. La evaluación pretratamiento de cada participante se lleva a cabo de manera individual por psicólogos entrenados en el protocolo de evaluación e intervención psicológica para maltratadores, donde se procede a la cumplimentación de cuestionarios y test estandarizados. Los participantes del programa firman un consentimiento informado de utilización de los datos con fines científicos. En la última sesión del Programa se realiza una evaluación postratamiento en la que se procede al pase de cuestionarios y test estandarizados de forma individual a los participantes que han finalizado el proceso de intervención.

Los análisis estadísticos de este estudio se han realizado con el paquete estadístico SPSS (versión 17.0 para Windows).

\section{Resultados}

Para evaluar si el programa produce cambios en las diferentes variables psicológicas en las que teóricamente pretende incidir, se ha llevado a cabo un contraste de medias pretratamiento y postratamiento en aquellas variables susceptibles de cambio.

\section{Cambios en actitudes hacia la violencia de género}

En la Tabla 2 se presentan las medias, las desviaciones típicas y pruebas $t$ de las Actitudes hacia la Violencia contra la Mujer en tiempo 1 (pretratamiento) y tiempo 2 (postratamiento). 
Tabla 2.

Resultados en las variables Actitudes hacia la Violencia contra la Mujer $(N=109)$.

\begin{tabular}{|c|c|c|c|c|}
\hline & Media & $\mathrm{N}$ & DT & $\mathrm{t}$ \\
\hline Gravedad T1 & 8.3041 & 97 & .18667 & \\
\hline Gravedad T2 & 8.7990 & 97 & .18407 & $-2.282^{*}$ \\
\hline Frecuencia T1 & 3.1275 & 102 & .71299 & \\
\hline Frecuencia T2 & 3.2647 & 102 & .76981 & -1.538 \\
\hline Culpa Víctima T1 & 2.9412 & 102 & 1.20085 & \\
\hline Culpa Víctima T2 & 2.6569 & 102 & 1.17309 & $2.007^{*}$ \\
\hline Aceptabilidad T1 & 1.5882 & 102 & .72239 & \\
\hline Aceptabilidad T2 & 1.5392 & 102 & .79193 & .528 \\
\hline Tolerancia T1 & 1.7451 & 102 & .85233 & \\
\hline Tolerancia T2 & 1.3431 & 102 & .63706 & $5.002^{* * *}$ \\
\hline Int. Denuncia T1 & 2.1863 & 102 & 1.21647 & \\
\hline Int. Denuncia T2 & 1.8725 & 102 & 1.19132 & $2.275^{*}$ \\
\hline
\end{tabular}

Como puede observarse, cuando se analiza si las diferencias observadas son significativas, se comprueba que esto es así para la Gravedad Percibida $\left(t_{96}=-2.282 ; p<.025\right)$, Culpabilización de la Víctima $\left(t_{101}=2.007 ; p<.047\right)$, Tolerancia $\left(t_{101}=5.002 ; p<.000\right)$, e Intención de Denuncia $\left(t_{101}=2.275 ; p<.025\right)$. No se observan diferencias significativas en Frecuencia $\left(t_{101}=-1.538 ; p<.127\right)$ y Aceptabilidad $\left(t_{101}=.528 ; p<\right.$ $.599)$. 
En relación a las variables relativas a la Atribución de Responsabilidad y Minimización, los resultados obtenidos en la comparación entre el pre y el postratamiento se representan en la Tabla 3. Como puede observarse, los resultados parecen indicar que tras el programa no aparecen diferencias significativas en las dimensiones Defensa Propia $\left(t_{92}=-1.683 ; p\right.$ $<.096)$ y Auto-atribución de Culpa $\left(t_{93}=.555 ; p<.580\right)$ y tampoco en la escala de Minimización $\left(t_{93}=1.392 ; p<.167\right)$. Únicamente la dimensión Culpabilización de la Víctima $\left(t_{93}=3.424 ; p<.001\right)$ presenta diferencia estadísticamente significativa.

Tabla 3.

Resultados en las variables Atribución de Responsabilidad y Minimización $(N=109)$.

\begin{tabular}{lrrcc}
\hline & Media & $\mathrm{N}$ & $\mathrm{DT}$ & $\mathrm{t}$ \\
\hline C. Víctima T1 & 59.1418 & 94 & 32.28874 & \\
C. Víctima T2 & 47.5780 & 94 & 33.64244 & $3.424^{* * *}$ \\
& & & & \\
Defensa Propia T1 & 33.3333 & 93 & 37.26574 & \\
Defensa Propia T2 & 39.9355 & 93 & 34.02518 & -1.683 \\
& & & & \\
Auto-atribuc. Culpa T1 & 20.9220 & 94 & 24.57828 & \\
Auto-atribuc. Culpa T2 & 19.5780 & 94 & 20.95829 & .555 \\
& & & & \\
Minimización T1 & 30.0319 & 94 & 22.21889 & \\
Minimización T2 & 26.6170 & 94 & 23.06675 & 1.392 \\
& & & & \\
\hline *** p <.001 & & & & \\
& & &
\end{tabular}


Cambios en las Variables de Ajuste Psicológico

Respecto al efecto del programa en la variable sintomatología depresiva, los resultados muestran un efecto significativo $\left(t_{106}=2.627 ; p=\right.$ .010). Los participantes han disminuido de manera significativa su sintomatología depresiva tras la intervención (Tabla 4). También se comprueba que no se encuentran diferencias significativas respecto a la Autoestima y a sus dimensiones, Autoestima general $\left(t_{76}=.562 ; p=.576\right)$, Autoestima Familiar $\left(t_{107}=.988 ; p=.326\right)$ y Autoestima Social $\left(t_{107}=-.573 ; p=.568\right)$.

Tabla 4.

Resultados en las variables Sintomatología Depresiva y Autoestima ( $N=109)$.

\begin{tabular}{lllll}
\hline Media & N & DT & t \\
\hline
\end{tabular}

\begin{tabular}{llll}
\hline S. Depresiva T1 & 13.1776 & 107 & 5.10607
\end{tabular}

S. Depresiva T2 $11.9439 \quad 107 \quad 4.53255 \quad 2.627^{* *}$

\begin{tabular}{llll}
\hline A. General T1 & 32.2078 & 77 & 4.18436
\end{tabular}
A. General T2
31.9351
$77 \quad 3.88769$
.562

A. Familiar T1 $12.4444 \quad 108 \quad 2.65926$
A. Familiar T2
12.1574
108
2.73490
.988
A. Social T1
11.7870
108
2.75834

$\begin{array}{lllll}\text { A. Social T2 } & 11.9630 & 108 & 2.29964 & -.573\end{array}$

${ }^{* *} \mathrm{p}<.01$ 


\section{Cambios en las Variables Contextuales}

La Tabla 5 recoge los resultados para las variables contextuales: Integración, Participación, Apoyo informal y Apoyo formal. Con respecto al efecto del programa sobre el Apoyo Social Comunitario, los resultados de la prueba $t$ de muestras relacionadas mostraron un efecto significativo para la variable Participación $\left(t_{106}=-2.820 ; p<.006\right)$. Además los resultados parecen indicar que tras el programa no hay diferencias significativas en las dimensiones Integración $\left(t_{106}=-.408 ; p=.684\right)$, Apoyo informal $\left(t_{104}=\right.$ $-1.321 ; p=.189)$ y Apoyo formal $\left(t_{104}=-.029 ; p=.977\right)$.

Tabla 5.

Resultados en las variables de Apoyo Social Comunitario $(N=109)$.

\begin{tabular}{lllll}
\hline & Media & $\mathrm{N}$ & $\mathrm{DT}$ & $\mathrm{t}$ \\
\hline Integración T1 & 10.7383 & 107 & 2.72423 & \\
Integración T2 & 10.8318 & 107 & 2.35318 & -.408 \\
Participación T1 & 11.4673 & 107 & 4.10319 & \\
Participación T2 & 12.6355 & 107 & 3.91753 & $-2.820^{* *}$ \\
A. Informal T1 & 18.2476 & 105 & 4.60096 & \\
A. Informal T2 & 18.9143 & 105 & 4.08354 & -1.321 \\
A. Formal T1 & 11.5048 & 105 & 2.81863 & \\
A. Formal T2 & 11.5143 & 105 & 2.72846 & -.029 \\
\hline ** $<.01$ & & & & \\
\hline
\end{tabular}




\section{Discusión y conclusiones}

El objetivo de este trabajo era evaluar si se producen cambios significativos como consecuencia de la implementación del Programa Contexto en un conjunto de variables psicosociales relevantes en términos de su consideración como factores protectores/de riesgo en relación a la violencia contra la mujer en las relaciones de pareja. Para este objetivo, se han planteado cuatro hipótesis de trabajo.

La primera hipótesis hacía referencia a que la intervención producirá cambios en actitudes relacionadas con la percepción de la violencia de género. Se observa en primer lugar, un aumento significativo en nuestra muestra en cuanto a la Gravedad Percibida de las situaciones de violencia de género. Este hecho podría estar indicando que los participantes, al finalizar el programa, son más conscientes de que la violencia de género no solamente es aquella violencia más cruel o reiterada y que hayan entendido que cualquier tipo de violencia, sea cual sea, es grave. Además, los participantes, tras la intervención, presentan menores niveles de Tolerancia hacia la violencia. Hay un cambio hacia un clima menos favorable hacia la violencia contra la mujer. Por otra parte, también se detecta, tras la intervención, un aumento en cuanto a la Intención de Denuncia en casos de violencia de género, lo que estaría sugiriendo una menor tolerancia hacia este tipo de violencia (Gracia \& Herrero, 2006a; Loseke \& Gelles, 1993; Muehlenhard \& Kimes, 1999). Todos estos cambios actitudinales podrían ser indicadores de una reconceptualización de las relaciones de pareja y de la utilización de la violencia como forma de resolver el conflicto, elemento imprescindible para disminuir la reincidencia. A pesar de que no se encuentran diferencias significativas en Frecuencia Percibida y Aceptabilidad de la violencia contra la mujer, los resultados muestran cambios en el sentido esperado. Esto sugiere la necesidad de trabajar más profundamente en aquellos grupos que mantienen estas actitudes, para lograr una prevención más eficaz (Gracia, 2004).

La segunda hipótesis planteaba que la intervención producirá cambios en los estilos de Atribución de Responsabilidad de los penados, de forma que se producirán más atribuciones relacionadas con la asun- 
ción de responsabilidad por sus propios actos y menos atribuciones que sitúen la culpa en terceras personas (la víctima) o agentes externos (la ley, la sociedad, el alcohol, etc.). Los resultados permiten afirmar que se produce una disminución en la Culpabilización de la Víctima. Este hecho supone que el participante, tras la intervención, llegue a ser consciente de una de las premisas básicas necesarias para el cambio: que él es parte responsable de la situación que le ha llevado a ser condenado. El hecho de que empiecen a asumir su responsabilidad respecto a la violencia será un factor clave de motivación para el cambio y un importante factor protector. Son numerosos los autores que señalan que estos cambios actitudinales $\mathrm{y}$ atribucionales se traducen en menores probabilidades de reincidencia en el futuro por parte del maltratador (Grann \& Wedin, 2002; Hanson \& Wallace-Capretta, 2000; Kropp \& Hart, 2000). En las variables Defensa Propia, Auto-atribución de Culpa y Minimización también se han producido cambios en la dirección esperada, pero no diferencias significativas. Es importante seguir profundizando en este tema, ya que tales distorsiones pueden hacer que estén menos motivados para el cambio y además puede suponer un mayor riesgo de reincidencia (Henning \& Holdford, 2006). Además el hecho que acudan por vía judicial, supone una motivación mucho menor y más resistencia al cambio (Echeburúa et al., 2006; Echeburúa \& Fernández-Montalvo, 2009; Fernández-Montalvo, Echeburúa \& Amor, 2005).

En relación a la tercera hipótesis, la intervención producirá cambios en variables de ajuste psicológico (sintomatología depresiva y autoestima). Los resultados indican que, tras la intervención, se reducen los niveles de sintomatología depresiva de los participantes. Este resultado es relevante si tenemos en cuenta que, a través de la intervención, se está reduciendo un factor de riesgo asociado reiteradamente con las características de los maltratadores (Maiuro et al., 1988). No podemos decir lo mismo en cuanto a la autoestima. El análisis no señala cambios en los niveles de la autoestima de los participantes tras la intervención. Respecto a ello, se observan resultados dispares en la literatura científica; mientras la mayoría de los estudios indica puntuaciones más bajas en autoestima en este tipo de población (Goldstein \& Rosenbaum, 1985; Steel \& Pollack, 1974; Walker, 1984), 
algunos trabajos muestran puntuaciones más elevadas (Jiménez, Musitu \& Murgui, 2008; Musitu \& Herrero, 2003; O’Moore \& Kirkham, 2001). En ambos casos, tanto la baja como la excesiva autoestima pueden suponer un potencial factor de riesgo para el desarrollo de problemas de conducta de carácter externalizante como la conducta violenta, por lo que en el proceso de intervención grupal el objetivo fundamental no es elevar ni disminuir la autoestima sino buscar un equilibrio. Esto puede ser la causa de que no sean visibles los cambios que hayan podido producirse en esta variable. Se requiere de futuros estudios que analicen los cambios en la autoestima de manera más pormenorizada.

La última hipótesis señalaba que la intervención producirá cambios en el apoyo social comunitario percibido de los penados. Como se observa en los resultados obtenidos, la intervención únicamente ha producido cambios significativos en el apoyo social comunitario percibido de los penados aumentando los niveles de participación. Este aumento podría indicar que, gracias a la intervención, hay un intento del propio participante, de forma activa, de conectarse a su comunidad, queriendo formar parte de ella. Este dato, de gran relevancia, es indicador de un efecto de cambio muy importante, el aumento de un factor protector para los participantes, a la vez que para los profesionales supone un dato que sigue corroborando la eficacia de la intervención (Antonovsky, 1979; Moos, 2005; Ortiz-Torres, 1999; Turner \& Turner, 1999; Zimmerman, 2000). No se han observado diferencias significativas en las variables Integración, Apoyo Informal y Apoyo Formal, pero sí cambios que indican una mejora de estas variables tras la finalización del programa. Es necesario seguir trabajando en esta dirección, conectando al participante a la sociedad, intentando centrar la atención en su red social y en los recursos sociales existentes que pueda aprovechar para intentar paliar problemáticas que le hagan más sensible hacia la violencia (Gracia \& Herrero, 2006b).

Todos estos resultados sirven como indicadores del grado de cambio (y, en cierto sentido, de eficacia) logrado con el programa.

El estudio que se ha realizado presenta también algunas limitaciones. En este sentido, sería conveniente para la mejora del programa y de cara a futuras investigaciones, solventar algunas debilidades, como es 
que se aumente la muestra del estudio, se tenga en cuenta que la utilización de un diseño preexperimental con medición pre y post tratamiento impide que se pueda asegurar que los cambios producidos sean debido a la intervención, a otro tipo de intervención o a factores que no se pueden controlar. Por otra parte, el tipo de muestra con la que se trabaja, por mandato judicial, puede influir en los resultados, dada la posible influencia de la deseabilidad social en los sujetos. También es difícil recoger información acerca de la reincidencia y además existen pocos datos que ofrezcan información de múltiples fuentes. Hay que ser prudentes a la hora de interpretar los resultados de las escalas pese a su moderada consistencia interna. Por tanto, a pesar de ver que los resultados nos ofrecen datos prometedores de cambios significativos en algunas variables importantes, son necesarios más estudios que permitan un análisis más profundo de la información disponible y, en base a ella, diseñar acciones certeras tendientes a la prevención y erradicación de este problema.

\section{Referencias}

Arce, R. \& Fariña, F. (2010). Diseño e implementación del Programa Galicia de Reeducación de Maltratadores: una respuesta psicosocial a una necesidad social y penitenciaria. Intervención Psicosocial, 19, 153-166, DOI: 10.593/in2010v19n2a7.

Antonovsky, A. (1979). Health, stress and coping. New perspectives on mental and physical well-being. San Francisco: Jossey-Bass.

Babcock, J. C. \& LaTaillade, J. (2000). Evaluating interventions for men who batter. En J. Vincent \& E. Jouriles (Eds.). Domestic violence: Guidelines for research-informed practice (pp. 37-77). Philadelphia: Jessica Kingsley.

Babcock, J. C., Green, C. E. \& Robie, C. (2004). Does batterer's treatment work? A metanalytic review of domestic violence treatment. Clinical Psychology Review, 23, 1023-1053.

Bennet, L. \& Williams, O. J. (2001). Controversies and recent studies of batterer intervention program effectiveness. Pennsylvania: National Online Resource Center on Violence Against Women.

Bronfenbrenner, U. (1979). The experimental ecology of human development. Cambridge: Harvard University Press. 
Cascardi, M. \& O'Leary, K. D. (1992). Depressive symptomatology, selfesteem and self-blame in battered women. Journal of Family Violence, 7, 249-259.

Chen, H., Bersani, C., Myers, S. \& Denton, R. (1989). Evaluating the effectiveness of a court sponsored abuser treatment program. Journal of Family Violence, 4, 309-322.

Cohen, S., Gottlieb, B. H. \& Underwood, L. G. (2000). Social relationships and health. En S. Cohen, L. G. Underwood \& B. H. Gottlieb (Eds.) Social support measurement and intervention: A guide for health and social scientists (pp. 3-25). Oxford: Oxford University Press.

Comisión Europea. (1999). Europeans and their views on domestic violence against women (Eurobarometer 51.0). Bruselas: Autor.

Coulter, M. \& VandeWeerd, C. (2009). Reducing domestic violence and other criminal recidivism: Effectiveness of a multi-level batterers intervention program. Violence and victims, 24(2), 139-153.

Cowen, E. L. (2000). Community psychology and routes to psychological wellness. En J. Rappaport \& E. Seidman (Eds.). Handbook of community psychology (pp. 79-99). Nueva York: Kluwer/Plenum.

Cranwell, M., Kolodinsky J., Carsten G., Schmidt F., Larson M. \& MacLachlan C. (2007). Short term change in attitude and motivating factors to change abusive behavior of male batterers after participating in a group intervention program based on the pro-feminist and cognitive-behavioral approach. Journal of Family Violence 2(2), 91-100.

Daly, J. \& Pelowski, S. (2000). Predictors of dropout among men who batter: Are view of studies with implications for research and practice. Violence and Victims, 15, 137-160.

Dunford, F. W. (2000). The San Diego Navy experiment: An assessment of interventions for men who assault their wives. Journal of Consulting and Clinical Psychology, 68, 468-476.

Echeburúa, E. \& Corral, P. (1998). Manual de violencia familiar. Madrid: Siglo XXI.

Echeburúa, E. \& Fernández-Montalvo, J. (2009). Evaluación de un programa de tratamiento en prisión de hombres condenados por violencia grave contra la pareja. International Journal of Clinical and Health Psychology, 9(1), 5-20.

Echeburúa, E., Fernández-Montalvo, J. \& Amor, P. J. (2006). Psychological treatment of men convicted of gender violence: A pilot-study in the Spanish prisons. International Journal of Offender Therapy and Comparative Criminology, 50, 57-70. 
Echeburúa, E., Sarasua, B., Zubizarreta, I. \& Corral, P. (2009). Evaluación de la eficacia de un tratamiento cognitivo-conductual para hombres violentos contra la pareja en un marco comunitario: una experiencia de 10 años (1997-2007). International Journal of Clinical and Health Psychology, 9, 199-217.

Eckersley, R., Dixon, J. \& Douglas, B. (2001). The social origins of health and well-being. Nueva York: Cambridge University Press.

Eckhardt, C. I., Murphy, C., Black, D. \& Suhr, L. (2006). Intervention programs for perpetrators of intimate partner violence: conclusions from a clinical research perspective. Public Health Reports, 121, 369381.

Feder, L. \& Forde, D. (2000). A test of the efficacy of court-mandated counseling for domestic violence offenders: The broward experiment. Final report to the U.S. Department of Justice, National Institute of Justice, NCJ 184752.

Feder, L. \& Wilson, D. B. (2005). A meta-analytic review of court-mandated batterer intervention programs: Can courts affect abusers' behavior? Journal of Experimental Criminology, 1, 239-262.

Feder, L. Wilson, D. B. \& Austin, S. (2008). Court-mandated interventions for individual convicted of domestic violence. Campbell Systematic Reviews, 12.

Fernández-Montalvo, J., Echeburúa, E. \& Amor, P.J. (2005). Aggressors against women in prison and in community: An exploratory study of a differential profile. International Journal of Offender Therapy and Comparative Criminology, 49, 158-167.

Ford, D. A. \& Regoli, M. J. (1993). The criminal prosecution of wife assaulters: Process, problems, and effects. En N. Zoe Hilton (Ed.). Legal responses to wife assault: Current trends and evaluation (pp. 127164). Newbury Park: Sage.

Goldstein, D. \& Rosenbaum, A. (1985). An evaluation of the self-steem of maritally violent men. Family relations, 34, 457-471.

Gondolf, A. (1987). Evaluating programs for men who batter: Problems and prospects. Journal of Family Violence, 2, 95-108.

Gondolf, E. W. (2002). Batterer intervention systems. Issues, outcomes, and recommendations. Thousand Oaks: Sage.

Gracia, E. (1997). El apoyo social en la intervención comunitaria. Barcelona: Paidós.

Gracia, E. (2004). Unreported cases of domestic violence against women: Towards an epidemiology of social silence, tolerance, and inhibition. Journal of Epidemiology and Community Health, 58, 536-537. 
Gracia, E. \& Herrero J. (2006a). Public attitudes toward reporting partner violence against women and reporting behavior. Journal of Marriage and the Family, 68, 759-768.

Gracia, E. \& Herrero, J. (2006b). Acceptability of domestic violence against women in the European Union: A multilevel analysis. Journal of Epidemiolical and Community Health, 60, 123-129.

Gracia, E., Herrero, J. \& Musitu, G. (2002). Evaluación de recursos y estresores psicosociales en la comunidad. Madrid: Síntesis.

Gracia, E., García, F. \& Lila, M. (2008). Police involvement in cases of intimate partner violence against women. Violence Against Women, 14, 697-714.

Gracia, E., Herrero, J., Lila, M. \& Fuente, A. (2009). Perceived neighborhood social disorder and attitudes toward domestic violence against women among latin-american immigrants. The European Journal of Psychology Applied to Legal Context, 1, 25-43.

Gracia, E., Herrero, J., Lila, M. \& Fuente, A. (2010). Percepciones y actitudes hacia la violencia de pareja contra la mujer en inmigrantes latinoamericanos en Espańa. Intervención Psicosocial, 19, 135-144, DOI: 10.5093/in2010v19n2a5.

Grann, M. \& Wedin, M. (2002). Risk factors for recidivism among spousal assault and spousal homicide offenders. Psychology, Crime \& Law, 8, 5-23.

Hanson, R. K. \& Wallace-Capretta, S. (2000). A multi-site study of treatment for abusive men (User Report No. 2000-05). Ottawa: Solicitor General Canada.

Henning, K. \& Holdford, R. (2006). Minimization, denial, and victim blaming by batterers: How much does the truth matter? Criminal Justice and Behavior, 33, 110-130.

Herrero, J. \& Gracia, E. (2007). Una medida breve de la sintomatología depresiva (CESD 7). Salud Mental 30(5), 40-46.

Jiménez, T. I., Musitu, G. \& Murgui, S. (2008). Funcionamiento familiar y consumo de sustancias en adolescentes: El rol mediador de la autoestima. International Journal of Clinic and Health Psychology, 8, 139-151.

Kropp, P. R. \& Hart, S. D. (2000). The Spousal Assault Risk Assessment (SARA) Guide: Reliability and validity in adult male offenders. Law and Human Behavior, 24, 101-118.

Ley Orgánica 1/2004, de 28 de diciembre, de Medidas de Protección Integral contra la Violencia de Género. B.O.E. núm. 313, de 29 de diciembre de 2004. http://www.mtas.es/mujer/violencia/ley/index.htm 
Lila, M., Herrero, J. \& Gracia, E. (2008). Evaluating attribution of responsibility and minimization by male batterers: implications for batterer programs. The Open Criminology Journal, 1, 4-11.

Lorente, M. (2001). Mi marido me pega lo normal. Barcelona: Editorial Planeta.

Loseke, D. R. \& Gelles, R. J. (1993). Conclusion: social problems, social policy, and controversies on family violence. En R. J. Gelles \& D. R. Loseke (Eds.). Current controversies on family violence (pp. 357-366). Newbury Park: Sage.

Maiuro, R. D., Cahn, T. S., Vitaliano, P. P., Wagner, B. C. \& Zegree, J. B. (1988). Anger, hostility, and depression in domestically violent versus generally assaultive men and nonviolent control subjectes. Journal of Consulting and Clinical Psychology, 56, 17-23.

Medina, J. (2002). Violencia contra la mujer: investigación comparada y situación en España. Valencia: Tirant lo Blanc.

Montero, M. (2003). Teoría y práctica de la psicología comunitaria: la tensión entre comunidad y sociedad. Barcelona: Paidós.

Moos, R. H. (2005). Contextos sociales, afrontamiento y bienestar: lo que sabemos y lo que necesitamos saber. Revista Mexicana de Psicología, 22, 15-30.

Muehlenhard, C. L. \& Kimes, L. A. (1999). The social construction of violence: The case of sexual and domestic violence. Personality and Social Psychology Review, 3, 234-245.

Musitu, G. \& Herrero, J. (2003). El rol de la autoestima en el consumo moderado de drogas en la adolescencia. Sociotam: Revista Internacional de Ciencias Sociales, 13, 285-306.

O’Moore, M. \& Kirkham, C. (2001). Self-esteem and its relationship to bullying behaviour. Aggressive Behavior, 27, 269-283.

Ortíz-Torres, B. (1999). El empowerment como alternativa teórica para la América Latina. Revista Interamericana de Psicología, 33, 49-66.

Radloff, L. S. (1977). The CES-D Scale: A self-report depression scale for research in the general population. Applied Psychological Measurement, 1, 385-401.

Real Academia Española, Diccionario en línea. Obtenido de http://www. rae.es/rae.html.

Rosenberg, M. (1965). Society and the adolescent self-image. Princeton: Princeton University Press.

Steel, B. F. \& Pollack, C. (1974). A psychiatric study of parents who abuse infants and small children. En R. Helfer \& C. Kempe (Eds.). The battered child (pp. 89-133). Chicago: Chicago University Press. 
Taylor, B., Davis, R. \& Maxwell, C. (2001). The effects of a group batterer programme: A randomized experiment in Brooklyn. Justice Quarterly, 18, 170-201.

Turner, R. J. \& Turner, J. B. (1999). Social integration and support. En C. C. Aneshensel, S. Carol \& J. C. Phelan (Eds.). Handbook of the sociology of mental health (pp. 301-319). Nueva York: Kluwer /Plenum.

Walker, L. (1984). The battered woman sindrome. New York: Springer.

Zimmerman, B. (2000). Attaining self-regulation. A social cognitive perspective. En M. Boekaerts, P. Pintrich \& M. Zeidner (Eds.). Handbook of self-regulation (pp. 13-35). California: Academic Press.

Fecha de recepción: 19 de julio de 2012.

Fecha de aceptación: 30 de noviembre de 2012. 